\title{
Gas in the Kidney (Emphysematous Pyelonephritis) often Misdiagnosed as Colonic Gas
}

MAZULKIFL $^{\mathrm{a}}$, MRALAM ${ }^{\mathrm{b}}$

of management. This can be achieved to a great extent by medical management combined with percutaneous drainage of obstructed kidney. However nephrectomy is indicated in life threatening infection of the kidney. Prompt diagnosis and the timing of drainage could be the prognostic factor. $A$ case of EPN in a diabetic patient who was successfully managed by nephrectomy is presented.

(J Banagladesh Coll Phys Surg 2015; 33: 91-94)

\section{Introduction:}

Emphysematous pyelonephritis (EPN) is defined as a severe, necrotizing renal parenchymal infection that is characterized by the bacterial production of gas within the kidney parenchyma. Schultz and Klorfein ${ }^{1}$ first used the term 'EPN' in 1962, but the condition might have already been described by Kelly and MacCullem ${ }^{1}$ as far back as the end of the previous century. EPN involves a spectrum of disease processes that result in the production of gas in the renal parenchyma; the gas can be focal or diffuse, and can spread to the collecting system or track into the perinephric and paranephric spaces. EPN can be classified into many ways and most of them are based on their CT findings. Middle-aged females with diabetes comprise the majority of patients with $\mathrm{EPN}^{2}$.

\section{Case Report:}

A 40 years old female diabetic (insulin dependent) was admitted into Square Hospital Ltd (SHL) through emergency room (ER) to the medical ward with

\footnotetext{
a. Dr. Mohammad Ali Zulkifl, Consultant, Urology, Square Hospital Ltd.

b. Dr. Mir. Rasekh Alam, Resident Medical Officer, Urology, Square Hospital Ltd

Address of Correspondence: Dr. Mohammad Ali Zulkifl, Consultant, Urology and Urological Surgeon, Square Hospital Ltd, 18/F, Bir Uttam Quzi Nuruzzaman sarak, West Panthapath, Dhaka 1205. Tel : 01711-345096, 01717-077700, Email : ovidmc@gmail.com
}

Received: 6 October, 2013

Accepted: 29 March, 2014

complaints of left sided abdominal pain, tightness of chest and decreased urine output for 7 days. There was no history of fever, cough or dysuria. She was admitted in a primary level hospital as a case of acute abdomen and treated conservatively for 3 days. As there was no improvement she was referred to a higher centre. On our medical ward she was treating as a patient of urosepsis with acute kidney injury. On the third day of admission we were called to see the incidentally diagnosed left renal stone. She had a plain X-ray kidney and urinary bladder (KUB) outside our hospital which was reported as normal except distended colonic gas shadow. By looking to the X-ray KUB with the diagnosis emphysematous Pyelonephritis (EPN) in mind we transferred her to urology department. Clinical evaluation revealed a co-operative but very ill patient. The temperature was $98 \mathrm{~F}$, pulse rate 88 per minute, blood pressure was 120/70 mm Hg and the respiratory rate 18 per minute. Cardio-vascular and respiratory systems were within normal limits. Abdominal examination revealed severe tenderness at the left upper abdomen and the left renal angle. There was no mass palpable, and the remainder of the physical examination was normal.

Laboratory investigations on admission showed a haemoglobin of $10.6 \mathrm{gm} / \mathrm{dl}$ (normal range: 11.5 to 16.5 $\mathrm{gm} / \mathrm{L}$ ), total leukocyte count of $11.5 \mathrm{~K} / \mu \mathrm{L}$ (normal 4.0 to $11 \mathrm{~K} / \mu \mathrm{L}$ ) with $88 \%$ neutrophils (normal 40 to $75 \%$ ). The platelet count was $99 \mathrm{~K} / \mu \mathrm{L}$ (normal 150 to 400 ). The the serum creatinine $2 \mathrm{mg} / \mathrm{dl}$ (normal range: 0.4 to $1.4 \mathrm{mg} / \mathrm{dl}$ ) 
and blood urea was $116 \mathrm{mg} / \mathrm{dl}$ (normal range : 35 to 40 $\mathrm{mg} / \mathrm{dl}$ ). The random blood sugar was $15 \mathrm{mmol} / \mathrm{L}$ (normal range 3.90 to $6.10 \mathrm{mmol} / \mathrm{L}$ ). The serum electrolyte was Sodium $130 \mathrm{mmol} / \mathrm{L}$, potassium $4.8 \mathrm{mmol} / \mathrm{L}$ (normal range : Sodium 135 to $145 \mathrm{mmol} / \mathrm{L}$ and potassium 3.5 to $4.5 \mathrm{mmol} / \mathrm{L}$ ). Urine microscopic examination revealed plenty pus cells, sugar $3+$ with trace amount of blood and ketones. Urine and blood cultures were sent and both showed no growth, possibly due to taking of antibiotic prior to admission. Blood group was B positive. CPR was $236.9 \mathrm{mg} / \mathrm{dl}$ (normal range $<5 \mathrm{mg} / \mathrm{dl}$ ).

A plain X-ray of the abdomen revealed kidney shaped gas in the left renal area (Fig. 1). Computerised tomography of the KUB confirmed the presence of gas in the renal and perirenal area with extensive renal parenchymal destruction (Fig. 2a and 2b) and pus tracking towards the left iliac fossa. The diagnosis was type II EPN.

The patient was initially treated with meropenem 500 mg 8 hourly. An emergency PCN was performed which immediately drained $100 \mathrm{ml}$ pus. As the patients condition was not improving expectedly left nephrectomy was done with left subcostal incision on the next day. Post operative period was otherwise uneventful except one day stay in ICU for delayed extubation. She was discharged on the $8^{\text {th }}$ POD with IV antibiotics for another 7 days. 6 months follow up was excellent.

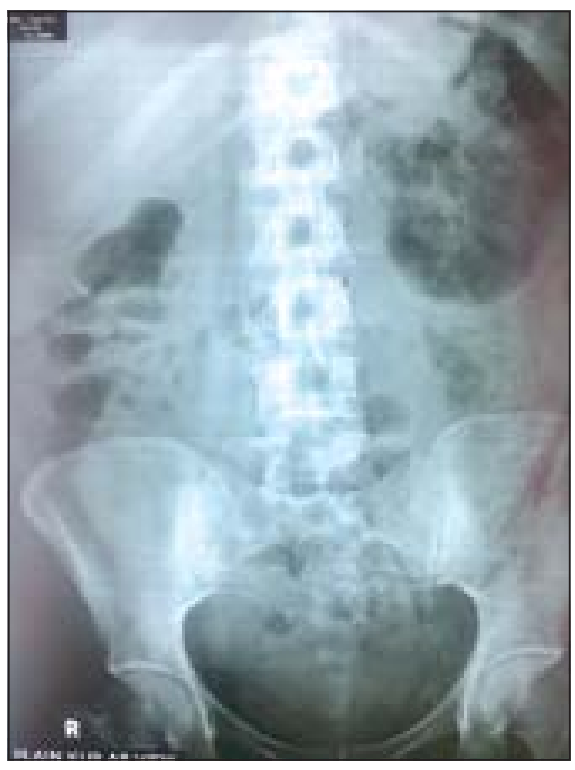

Fig.-1: X-ray KUB showing kidney shaped gas in left renal area

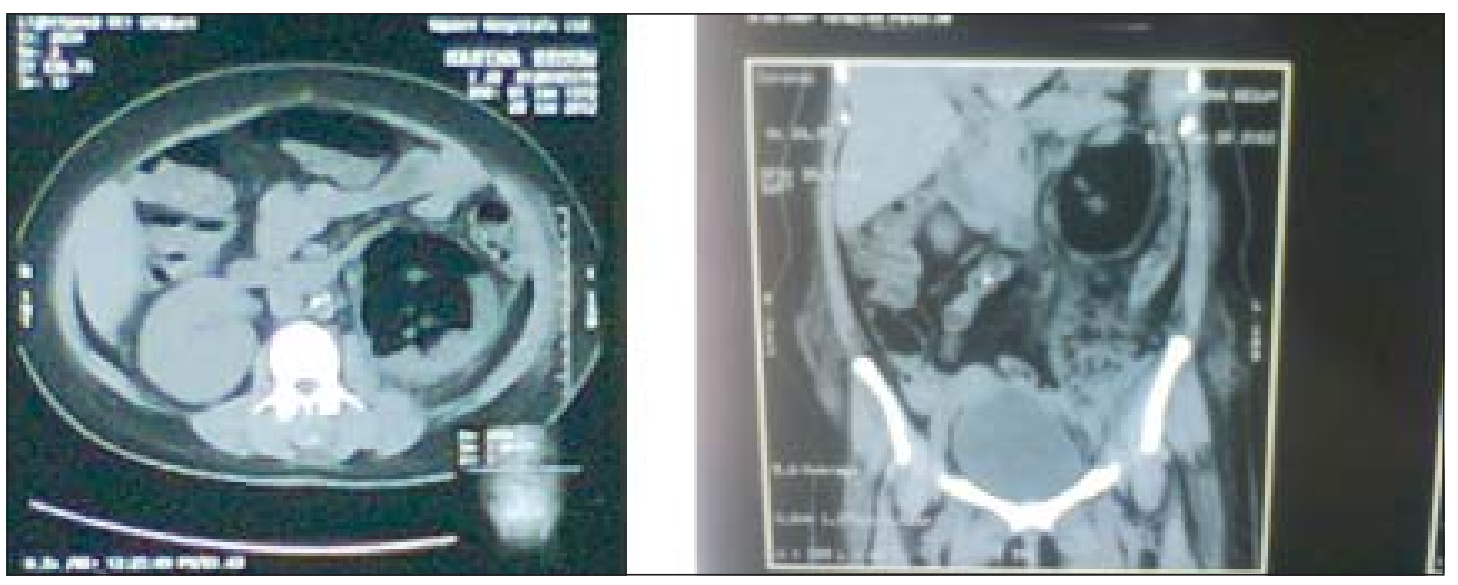

Fig.-2a and 2b: showing gas in left kidney and perirenal tissue 


\section{Discussion:}

EPN is a rare renal infection. However due to modern imaging techniques and practice of routine ultrasound more cases are reported now a day. EPN predominantly affects females. The female to male ratio is $3: 1$. The left kidney is more frequently involved than the right $(60 \%$ Vs $35 \%$ ). Both the kidneys are involved in about $5 \%$ of the reported series. $90 \%$ of the reported cases have occurred in diabetic patients. EPN has also been reported in debilitated (alcoholic) and immunocompromised patients. ${ }^{4}$

EPN can be classified into many ways and most of them are based on their CT findings. One of the classifications of EPN by Huang and Tseng ${ }^{2}$ (based on CT) is Class I: Gas in collecting system only. Class II: Parenchymal gas only. Class IIIa: Extension of gas into perinephric space. Class IIIb: Extension of gas into pararenal space. Class IV: EPN in solitary kidney, or bilateral disease. Another simple classification of EPN by Wan et $\mathrm{al}^{3}$ (based on CT) Type I: Renal necrosis with presence of gas but no fluid. Type II: Parenchymal gas associated with fluid in renal parenchyma, perinephric space or collecting system.

The organisms commonly responsible for causing EPN are Escherichia coli, Klebsiella pneumoniae, Proteus mirabilis, Pseudomonas aeruginosa, Citrobacter and rarely yeast. If left untreated the condition is uniformly fatal. ${ }^{45}$ The exact mechanism of gas formation in EPN is not known. Gas formation is believed to be due to pathogenic bacteria capable of mixed acid fermentation acting in a hyperglycaemic environment on tissues that are ischaemic. This results in tissue destruction, and encourages purulent infection and inhibition of the removal of locally produced gas $^{8}$

The clinical presentation of emphysematous pyelonephritis was similar to that of upper urinary tract infection with fever, nausea and vomiting, lethargy, confusion, dyspnoea and shock. Laboratory data showed high glycosylate haemoglobin, leukocytosis, thrombocytopenia and pyuria. ${ }^{3}$

Diagnosis of EPN rests on the clinical awareness and confirming it by appropriate investigations. The triad of symptoms of fever, flank pain and pyuria especially in diabetic patients who do not respond promptly to antibiotic treatment must raise the possibility of EPN. ${ }^{8}$ These patients require to be investigated and treated aggressively. The diagnosis of EPN is classically made by demonstrating gas in the renal or peri-renal tissue by plain abdominal X-ray. However gas can be demonstrated only in 33\% of plain abdominal radiographs in patients with EPN. ${ }^{6}$ Even by abdominal ultrasonography it may be technically difficult to distinguish the renal gas filled area from gas in the bowel. On the other hand, CT scan can not only confirm the diagnosis, but also show the extent of the disease. Abdominal CT scan is recommended for all patients in whom EPN is suspected.

Several studies have been done to correlate clinical features of EPN with the treatment outcome. ${ }^{7}$ These showed that age, sex, site of infection, blood urea nitrogen level and blood glucose level were not prognostic factors. But patients initially seen with thrombocytopenia, acute renal function impairment, disturbance of consciousness and shock were associated with very high mortality. ${ }^{8}$

Management of patients with emphysematous pyelonephritis has been a subject of controversy. Huang and Tseng reviewed the management of 48 patients with emphysematous pyelonephritis. They concluded that for localized emphysematous pyelonephritis (class I and II ) according to CT scan, percutaneous drainage with antibiotic treatment can provide a good outcome. For extensive emphysematous pyelonephritis (class III and IV ) with more benign manifestations, when saving the kidney is possible, percutaneous drainage combined with antibiotic treatment may be attempted because of its high success rate. However, nephrectomy can provide the best management outcome and should promptly be attempted for extensive emphysematous pyelonephritis with a fulminant course. ${ }^{3}$ The rapidly deteriorating general condition of the patient and the onset of septicaemic shock prompted us to go ahead with nephrectomy rather than adopt a more conservative line of management.

\section{Conclusion:}

EPN is a severe and often life threatening infection. CT Scan is the investigation of choice for not only making a proper diagnosis but also in planning the treatment option. Renal preservation must be the aim of treatment, but this must not be at the cost of patient's life. One should not hesitate to resort to nephrectomy as and when indicated. 


\section{References:}

1. Schultz E. H. Jr, Klorfein E.H. Emphysematous pyelonephritis. J. Urol. 1962;87: 762-766.

2. Kelly H. A., MacCullem W. G. Pneumaturia. JAMA 1898;31:375.

3. Huang JJ, Tseng CC. Emphysematous pyelonephritis: clinicoradiological classification, management, prognosis, and pathogenesis. Arch Intern Med. 2000;160:797-805.

4. Heinman S. Emphysematous Pyelonephritis. J Urol 1984; 131:203-208.

5. Alan R. Pontin, Richard D. Barnes. Nature Reviews Urology 2009;6:272-279 .

6. Wan Y. L., Lo S. K., Bullard M., Chong P. L., Lee T. Y. Predictors of outcome in emphysematous pyelonephritis. J. Urol. 1988;159:369-373

7. Andersen JB. Pyelonephritis emphysematosa. En alvorlig komplikation til diabetes mellitus. Ugeskr Laeger 1992; 1419-21.
8. Wang JM, Lim HK, Pang KK. Emphysematous pyelonephritis. Scand J Urol Nephro 2007;1:223-9.

9. Huang JJ, Chen KW, Ruaan MK. Mixed acid fermentation of glucose as a mechanism of emphysemtous urinary tract infection. J Urol 1991; 146:148-151.

10. Patterson JE, Andriole VT, Bacterial Urinary Tr a c t Infections In Diabetes. Infectious Disease Clinics of North America 1995; 9:25-51.

11. Heinman S. Emphysematous Pyelonephritis. J Urol 1984; 131:203-208.

12. Wan YL, Lee TY, Bullard MJ, Tsai CC. Acute gas producing bacterial renal infection: correlation between imaging findings and clinical outcome. Radiology 1996; 198:433-438.

13. Wan YL, Lo SK, Bullard MJ, Chang PL, Lee TY. Predictors of outcome in emphysematous pyelonephritis. J Urol 1998; 159:369-373. 